04.2

\title{
Влияние низкочастотных магнитогидродинамических мод на развитие филаментов в токамаке Глобус-М
}

\author{
(C) В.В. Буланин ${ }^{1,2}$, В.К. Гусев ${ }^{2}$, Г.С. Курскиев ${ }^{2}$, В.Б. Минаев ${ }^{2}$, М.И. Патров ${ }^{2}$, А.В. Петров ${ }^{1}$, \\ Ю.В. Петров ${ }^{2}$, Д.В. Присяжнюк ${ }^{3}$, Н.В. Сахаров ${ }^{2}$, В.В. Солоха ${ }^{2}$, \\ С.Ю. Толстяков ${ }^{2}$, Н.А. Хромов ${ }^{2}$, А.Ю. Яшин ${ }^{1,2}$
}

${ }^{1}$ Санкт-Петербургский политехнический университет Петра Великого, Санкт-Петербург, Россия

${ }^{2}$ Физико-технический институт им. А.Ф. Иофрфе РАН, Санкт-Петербург, Россия

${ }^{3}$ Институт ффизики плазмы им. Макса Планка, Гархинг, Германия

E-mail: V.Bulanin@spbstu.ru

Поступило в Редакцию 17 июня 2019r.

В окончательной редакции 17 июня 2019г.

Принято к публикации 18 июня 2019г.

\begin{abstract}
Представлены экспериментальные данные, свидетельствующие о влиянии магнитогидродинамической тиринг-моды в сферическом токамаке Глобус-М на возникновение нитевидных структур (филаментов). Регистрация филаментов осуществлялась методом допплеровского обратного рассеяния. Обнаружены группы филаментов, локализованные в тороидальном направлении, появление которых оказалось синхронизованным с распространением тиринг-моды. Рассматриваются возможные причины влияния низкочастотных магнитогидродинамических колебаний на возникновение групп нитевидных возмущений.
\end{abstract}

Ключевые слова: плазма, токамак, филаменты, тиринг-мода, допплеровское обратное рассеяние.

DOI: 10.21883/PJTF.2019.19.48312.17933

В настоящее время известно большое число экспериментальных исследований нитевидных структур, или филаментов, возникающих на периферии плазмы токамаков [1-4]. Эти возмущения плазмы, сильно вытянутые вдоль магнитного поля и локализованные в полоидальной плоскости, наблюдаются как в L-, так и в Н-моде $[5,6]$ в областях вблизи последней замкнутой магнитной поверхности [7]. Установлено, что в Н-моде достигаются условия, при которых филаменты возникают из-за возрастания градиента плазменного давления и увеличения плотности тока в области пьедестала, дестабилизирующих пилинг-баллонную неустойчивость [8-13]. В настоящей работе обнаружено, что нитевидные структуры могут возникать в токамаке при развитии сильной тиринг-моды, когда в плазме в областях, локализованных по полоидальному углу, периодически повышается градиент давления плазмы и, видимо, появляются условия для развития пилингбаллонной моды.

Группы нитевидных образований были обнаружены в сферическом токамаке Глобус-М $(\varepsilon=R / a=$ $=0.24 \mathrm{~m} / 0.36 \mathrm{~m}=0.66) \quad$ в дейтериевых разрядах $\mathrm{c}$ омическим нагревом при токе $I_{p}=190 \mathrm{kA}$ и магнитном поле $B_{T}=0.4$ Т. Была реализована магнитная конфигурация с одной $X$-точкой, расположенной в направлении тороидального дрейфа ионов. Временнь́е зависимости основных параметров представлены на рис. 1, $a$. В разрядах наблюдалась сильная магнитогидродинамическая (МГД) активность (рис. 1, b). С помощью магнитных измерений было установлено, что в плазме в окрестности запаса устойчивости, равного $q=2(R=54 \mathrm{~cm}, 5 \mathrm{~cm}$ от сепаратрисы), развивается тиринг-мода с модовыми числами $m / n=2 / 1$. Характерная частота МГД-колебаний составляла $8 \mathrm{kHz}$, а амплитуда колебаний полоидальной компоненты магнитного поля, измеряемая магнитным зондом на радиусе $R=62 \mathrm{~cm}$, составляла $2.5 \cdot 10^{-3} \mathrm{~T}$.

Для регистрации филаментов использовался метод микроволнового допплеровского обратного рассеяния (ДОР). Метод активно используется для измерения скоростей вращения плазмы и колебаний скоростей вращения в тороидальных системах магнитного удержания [14-25]. Возможность регистрации филаментов с помощью метода ДОР была продемонстрирована впервые в работе [26]. Допплеровское обратное рассеяние на филаменте проявляется в появлении локализованного по времени (цуга) квазикогерентного сигнала квадратурного (IQ) детектора обратно рассеянного излучения. Частота этого сигнала $\omega$ определяется скоростью филамента $V-\omega=k V$, где $k-$ удвоенное волновое число зондирующего излучения в отсечке при наклонном падении СВЧ-пучка. Длительность цуга определяется временем прохождения филамента через объем рассеяния в полоидальном направлении.

Для приема обратно рассеянного излучения на токамаке использовалась схема с двойным гомодинным приемом. Работа схемы, определение величины волнового числа $k$ и радиуса отсечки для реальной геометрии сферического токамака детально описаны в работе [24]. Зондирование излучением О-моды осуществлялось в экваториальной плоскости токамака со стороны слабого магнитного поля. При частоте зондирующего излучения $37 \mathrm{GHz}$ и угле наклона падающего пучка в $12^{\circ}$ отсечка, 


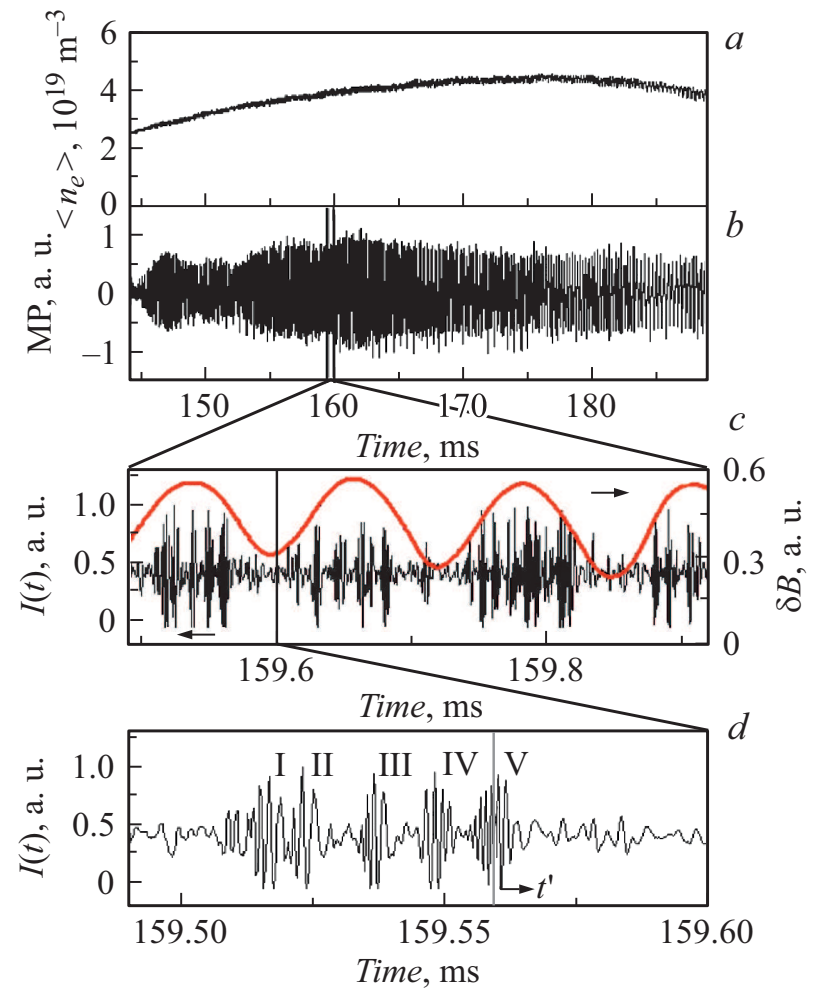

Рис. 1. Временнб́е зависимости: $a-$ средней плотности плазмы, $b-$ сигнала магнитного зонда, $c$ - сигнала ДОР (левая ось) и возмущений магнитного поля (правая ось), $d-$ сигнала ДОР с указанием номеров филаментов (римские цифры) и момента времени, для которого выполнена реконструкция на рис. 2 .

при которой происходит обратное рассеяние, находилась внутри последней замкнутой магнитной поверхности на радиусе $R=57 \mathrm{~cm}$ для временно́го интервала, представленного на рис. $1, c$.

Во время развития сильной МГД-активности было обнаружено, что цуги квазикогерентных колебаний (ЦКК), которые формируются при обратном рассеянии на филаментах, появляются группами. Пример такой регистрации для разряда \#28775 показан на рис. 1, c. Эти группы представляют собой последовательность 3-5 филаментов с временем регистрации одного филамента около 8-10 $\mu$ s. Между группами наблюдаются временны́е интервалы, в которых филаменты не наблюдались. Иными словами, филаменты были локализованы в тороидальном (полоидальном) направлении. Одна из таких групп филаментов подробно представлена на рис. $1, d$. Каждый из цугов квазикогерентных колебаний на рис. 1,d отражает обратное рассеяние на одном филаменте. Перпендикулярная скорость $V_{\perp}$ каждого филамента в группе определялась с помощью соотношения $\omega=k V_{\perp}$. По оценкам величины скорости определялось расстояние $S_{\perp}=V_{\perp} \Delta t$ между филаментами в направлении, перпендикулярном силовым линиям магнитного поля, и реконструировалось пространственное распределение филаментов. Пример такой реконструкции приведен на рис. 2, на котором ясно видна локализация филаментов в полоидальном и тороидальном направлениях. При известной величине $V_{\perp}$ можно было определить соответствующую тороидальную скорость $V_{T}=V_{\perp} / \sin \alpha$ ( $\alpha$ - угол наклона силовых линий магнитного поля) и квазитороидальное модовое число $n=2 \pi R /\langle\Delta t\rangle\left\langle V_{T}\right\rangle$ (здесь $\langle\Delta t\rangle-$ средний временной интервал между ЦКК, $R$ - большой радиус, на котором расположена отсечка). Для филаментов внутри групп средняя величина тороидального модового числа равнялась $n=15$.

Было обнаружено, что появление групп филаментов синхронизовано с МГД-колебаниями, которые регистрировались магнитными зондами. Это продемонстрировано на рис. 1,c, где помимо сигнала IQ-детектора показано колебание полоидальной компоненты магнитного поля, обусловленное развитием тиринг-моды. Сопоставление МГД-сигналов и групп ЦКК показало, что филаменты возникают в определенной фазе МГДколебаний, а именно когда магнитный остров находится на внешнем обходе тора (напротив антенны). Таким образом, локализация групп филаментов отвечает периодичности в тороидальном направлении с номером $n=1$, равным тороидальному модовому числу тирингмоды. В работе [27] было показано, что при данной тороидальной периодичности в условиях токамака ASDEXUpgrade наибольшим инкрементом обладают филаменты с модовым числом $n=13$. В нашем эксперименте это число равно 15. Такие группы филаментов во время МГД-активности возникали не всегда. Они наблюдались только при превышении некоторого порогового уровня МГД-колебаний, что иллюстрируется рис. $1, b$, на котором отмеченная область появления групп филаментов совпадает с областью повышенной МГД-активности в интервале от 150 до $165 \mathrm{~ms}$. Группы филаментов не

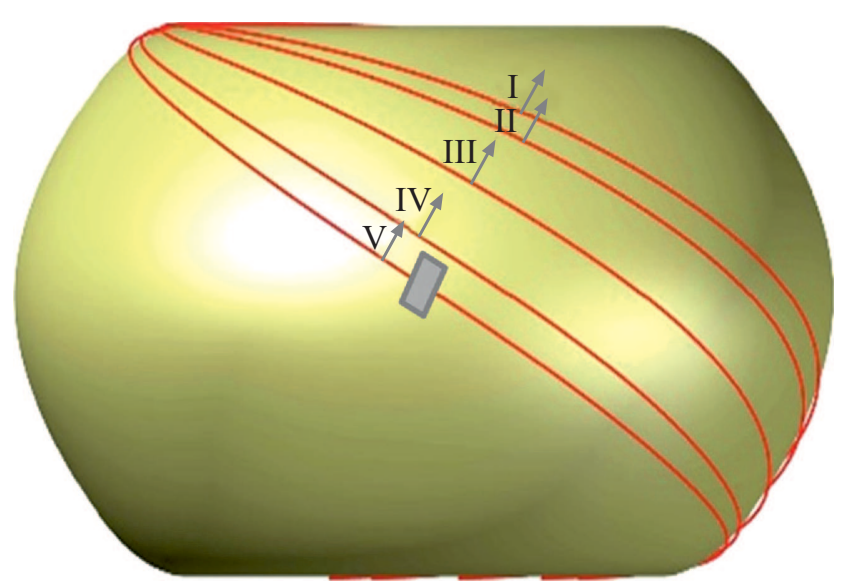

Рис. 2. Вид сбоку на магнитную поверхность токамака Глобус-М. Реконструкция расположения филаментов $(\mathrm{I}-\mathrm{V})$ в момент времени $t^{\prime}$ (см. рис. $1, d$ ). Филаменты условно показаны линиями, направленными вдоль силовых линий магнитного поля. Стрелками обозначены направления движения филаментов, прямоугольник - расположение приемной антенны ДОР. 


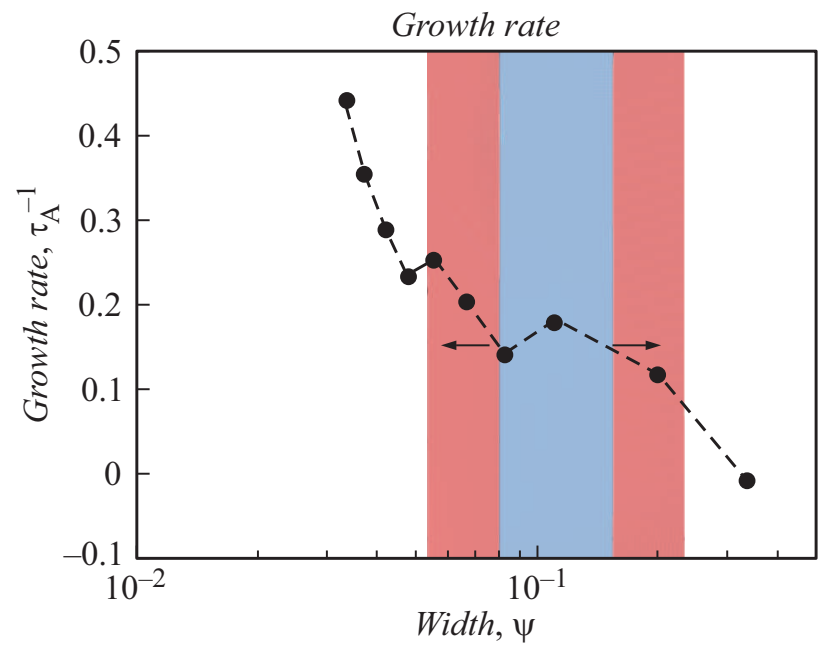

Рис. 3. Зависимость инкремента нарастания пилингбаллонной неустойчивости от ширины пьедестала. Инкремент представлен в единицах периода альфвеновских колебаний $\tau_{\mathrm{A}}$, рассчитанного для области развития филаментов. Полосы определяют ширину пьедестала как среднюю величину по всему квазистационарному периоду разряда от 150 до $165 \mathrm{~ms}$ (полоса в центре) и как среднюю величину по интервалам периода прохождения $O$ - и $X$-точек МГД-острова (полосы по краям).

наблюдались при смещении отсечки при изменении частоты зондирования.

Расположение области регистрации филаментов между центром острова при $q=2$ и сепаратрисой означает, что в области отсечки при прохождении острова должны возрастать давление плазмы и его градиент между отсечкой и сепаратрисой. В такой ситуации в локализованной области в полоидальном и тороидальном направлениях возможно появление филаментов. Их возникновение может быть обусловлено двумя причинами. Первая состоит в том, при МГД-колебаниях из-за возмущения плотности смещается отсечка, при этом она может оказаться как внутри области развития филаментов, так и вне ее. Однако, как показывают оценки, при наблюдаемых возмущениях плотности плазмы отсечка смещается на расстояние, значительно меньшее, чем радиальное разрешение метода (около $1 \mathrm{~cm}$ ). В этом случае нельзя ожидать чередований в появлении и исчезновении филаментов. Вторая причина может быть связана с тем, что градиент давления в исследуемой области изменяется при прохождении острова. Увеличение градиента давления, обусловленное прохождением О-точки магнитного острова на внешнем обходе тора, может приводить к временному увеличению инкремента возрастания баллонной неустойчивости и возникновению серии филаментов.

Для подтверждения этого предположения было проведено моделирование с использованием кода $\mathrm{BOUT}++[27,28]$ для условий токамака Глобус-М. Полагалось, что ток проводимости меняется слабо на пери- ферии плазмы. Это позволило определить зависимость инкремента пилинг-баллонной моды только от одного параметра (ширины пьедестала), который определяет градиент давления и бутстреп-ток. Этот параметр оценивался как показатель степени гиперболического тангенса, которым аппроксимировались профили плазмы, определенные методом томсоновского рассеяния. При этом высота пьедестала устанавливалась в квазистационарных условиях разряда в период от 150 до $165 \mathrm{~ms}$ равной 9 mbar. Ширина пьедестала выражалась в долях полоидального магнитного потока в токамаке - $\psi$. Пример расчета инкремента пилинг-баллонной моды для разряда \#28775 представлен на рис. 3. Полосой в центре показан интервал изменения средней ширины пьедестала. Ширина этого интервала определялась незначительным изменением средней плотности плазмы. Полосы по краям определяют зоны усредненной ширины пьедестала, определенные в периоды прохождения $O$ и $X$-точек мимо объема обратного рассеяния. Видно, что при крайних значениях ширины пьедестала инкремент, выраженный в периодах альфвеновской частоты, меняется от 0.05 до 0.25. При малых значениях инкремента неустойчивость может не развиться за полпериода МГД-колебания, а при больших значениях развитие неустойчивости и соответственно появление филаментов возможно. Для подтверждения этой интерпретации требуется проведение измерений периферийных плазменных параметров с большей точностью, и прежде всего более точная оценка тока проводимости.

В заключение отметим, что на возможную нелинейную связь между локализованными в тороидальном и полоидальном направлениях нитевидными возмущениями и тиринг-модой было указано в работе [29]. В экспериментах на токамаке DIII-D связь между этими типами колебаний была обнаружена, только в обратном по сравнению с нашими экспериментами направлении: развитие периферийной локализованной моды (ELM) приводило к возбуждению тиринг-моды [30,31].

\section{Финансирование работы}

Исследование выполнено за счет гранта Российского научного фонда (проект № 18-72-10028).

\section{Конфликт интересов}

Авторы заявляют, что у них нет конфликта интересов.

\section{Список литературы}

[1] Kirk A., Wilson H.R., Counsell G.F., Akers R., Arends E., Cowley S.C., Dowling J., Lloyd B., Price M., Walsh M. // Phys. Rev. Lett. 2004. V. 92. N 24. P. 245002.

[2] Vicente J., Conway G.D., Manso M.E., Müller H.W., Silva C., da Silva F., Guimarãis L., Silva A. // Plasma Phys. Control. Fusion. 2014. V. 56. N 12. P. 125019. 
[3] Yun G.S., Lee W., Choi M.J., Lee J., Park H.K., Tobias B., Domier C.W., Luhmann N.C., Jr., Donné A.J.H., Lee J.H. // Phys. Rev. Lett. 2011. V. 107. N 4. P. 045004.

[4] Ben Ayed N., Kirk A., Dudson B., Tallents S., Vann R.G.L., Wilson H.R. and the MAST team // Plasma Phys. Control. Fusion. 2009. V. 51. N 3. P. 035016.

[5] Kirk A., Ben Ayed N., Counsell G., Dudson B., Eich T., Herrmann A., Koch B., Martin R., Meakins A., Saarelma S., Scannell R., Tallents S., Walsh M., Wilson H.R. and the MAST team // Plasma Phys. Control. Fusion. 2006. V. 48. N 12B. P. B433-B441.

[6] Kirk A., Asakura N., Boedo J.A., Beurskens M., Counsell G.F., Eich T., Fundamenski W., Herrmann A., Kamada Y., Leonard A.W., Lisgo S., Loarte A., Oyama N., Pitts R.A., Schmid A., Wilson H.R. // J. Phys.: Conf. Ser. 2008. V. 123. P. 012011.

[7] Spolaore M., Kovařik K., Stöckel J., Adamek J., Dejarnac R., Ďuran I., Komm M., Markovic T., Martines E., Panek R., Seidl J., Vianello N., the COMPASS team // Nucl. Mater. Energy. 2017. V. 12. P. 844-851.

[8] Connor J.W., Hastie R.J., Wilson H.R. // Phys. Plasmas. 1998. V. 5. N 7. P. 2687-2700.

[9] Snyder P.B., Wilson H.R., Osborne T.H., Leonard A.W. // Plasma Phys. Control. Fusion. 2004. V. 46. N 5A. P. A131A141.

[10] Pankin A.Y., Bateman G., Brennan D.P., Schnack D.D., Snyder P.B., Voitsekhovitch I., Kritz A.H., Janeschitz G., Kruger S., Onjun T., Pacher G.W., Pacher H.D. // Nucl. Fusion. 2006. V. 46. N 4. P. 403-411.

[11] Medvedev S.Yu., Martynov A.A., Martin Y.R., Sauter O., Villard L. // Plasma Phys. Control. Fusion. 2006. V. 48. N 7. P. 927-938.

[12] Leonard A.W. // Phys. Plasmas. 2014. V. 21. N 9. P. 090501.

[13] Solokha V.V., Kurskiev G.S., Bulanin V.V., Petrov A.V., Tolstyakov S.Yu., Mukhin E.E., Gusev V.K., Petrov Yu.V., Sakharov N.V., Tokarev V.A., Khromov N.A., Patrov M.I., Bakharev N.N., Sladkomedova A.D., Telnova A.Yu., Shchegolev P.B., Kiselev E.O., Yashin A.Yu. // J. Phys.: Conf. Ser. 2018. V. 1094. P. 012002

[14] Bulanin V.V., Lebedev S.V., Levin L.S., Roytershteyn V.S. // Plasma Phys. Rep. 2000. V. 26. N 10. P. 813-819.

[15] Hirsch M., Holzhauer E., Baldzuhn J., Kurzan B., Scott B. // Plasma Phys. Control. Fusion. 2001. V. 43. N 12. P. 16411660 .

[16] Conway G.D., Scott B., Schirmer J., Reich M., Kendl A. and the ASDEX Upgrade team // Plasma Phys. Control. Fusion. 2005. V. 47. N 8. P. 1165-1186.

[17] Hennequin P., Honoré C., Truc A., Quémńeur A., FenziBonizec C., Bourdelle C., Garbet X., Hoang G.T. and the Tore Supra team // Nucl. Fusion. 2006. V. 46. N 9. P. S771S779.

[18] Schmitz L., Zeng L., Rhodes T.L., Hillesheim J.C., Doyle E.J., Groebner R.J., Peebles W.A., Burrell K.H., Wang G. // Phys. Rev. Lett. 2012. V. 108. N 15. P. 155002.

[19] Hillesheim J.C., Crocker N.A., Peebles W.A., Meyer H., Meakins A., Field A.R., Dunai D., Carr M., Hawkes N. and the MAST team // Nucl. Fusion. 2015. V. 55. N 7. P. 073024.

[20] Bulanin V.V., Gusev V.K., Iblyaminova A.D., Khromov N.A., Kurskiev G.S., Minaev V.B., Patrov M.I., Petrov A.V., Petrov Yu.V., Sakharov N.V., Shchegolev P.B., Tolstyakov S.Yu., Varfolomeev V.I., Wagner F., Yashin Yu. // Nucl. Fusion. 2016. V. 56. N 1. P. 016017.
[21] Zhong W.L., Shi Z.B., Xu Y., Zou X.L., Duan X.R., Chen W., Jiang M., Yang Z.C., Zhang B.Y., Shi P.W., Liu Z.T., Xu M., Song X.M., Cheng J., Ke R., Nie L., Cui Z.Y., Fu B.Z., Ding X.T., Dong J.Q., Liu Y., Yan L.W., Yang Q.W., Liu Y. and HL-2A team // Nucl. Fusion. 2015. V. 55. N 11. P. 113005.

[22] Zhou C., Liu A.D., Zhang X.H., Hu J.Q., Wang M.Y., Li H., Lan T., Xie J.L., Sun X., Ding W.X., Liu W.D., Yu C.X. // Rev. Sci. Instrum. 2013. V. 84. N 10. P. 103511.

[23] Bulanin V.V., Askinazi L.G., Belokurov A.A., Kornev V.A., Lebedev V., Petrov A.V., Tukachinsky A.S., Vildjunas M.I., Wagner F., Yashin A.Yu. // Plasma Phys. Control. Fusion. 2016. V. 58. N 4. P. 045006.

[24] Yashin A.Y., Bulanin V.V., Petrov A.V., Petrov M.A., Gusev V.K., Khromov N.A., Kurskiev G.S., Patrov M.I., Petrov Y.V., Tolstyakov S.Y., Prisyazhnyuk D.V. // JINST. 2015. V. 10. P. P10023.

[25] Буланин В.В., Гусев В.К., Курскиев Г.С., Минаев В.Б., Патров М.И., Петров А.В., Петров М.А., Петров Ю.В., Тельнова А.Ю., Яшин А.Ю. // Письма в ЖТФ. 2017. Т. 43. B. 23. C. $40-47$.

[26] Буланин В.В., Варфоломеев В.И., Гусев В.К., Иванов А.Е., Крикунов С.В., Курскиев Г.С., Ларионов М.М., Минаев В.Б., Патров М.И., Петров А.В., Петров Ю.В., Сахаров Н.В., Толстяков С.Ю., Хромов Н.А., Яшин А.Ю. // Письма в ЖТФ. 2011. Т. 37. В. 7. С. 103-110.

[27] Dudson B., Umansky M.V., Xu X.Q., Snyder P.B., Wilson H.R. // Comp. Phys. Commun. 2009. V. 180. N 9. P. $1467-1480$.

[28] Dudson B., Madsen J., Omotani J., Hill P., Easy L., Løiten M. // Phys. Plasmas. 2016. V. 23. N 6. P. 062303.

[29] Hölzl M., Günter S., Wenninger R.P., Müller W.-C., Huysmans G.T.A., Lackner K., Krebs I., and ASDEX Upgrade team // Phys. Plasmas. 2012. V. 19. N 8. P. 082505.

[30] Sauter O., La Haye R.J., Chang Z., Gates D.A., Kamada Y., Zohm H., Bondeson A., Boucher D., Callen J.D., Chu M.S., Gianakon T.A., Gruber O., Harvey R.W., Hegna C.C., Lao L.L., Monticello D.A., Perkins F., Pletzer A., Reiman A.H., Rosenbluth M., Strait E.J., Taylor T.S., Turnbull A.D., Waelbroeck F., Wesley J.C., Wilson H.R., Yoshino R. // Phys. Plasmas. 1997. V. 4. N 5. P. 1654-1664.

[31] Petty C.C., Austin M.E., Holcomb C.T., Jayakumar R.J., La Haye R.J., Luce T.C., Makowski M.A., Politzer P.A., Wade M.R. // Phys. Rev. Lett. 2009. V. 102. N 4. P. 045005. 